\title{
Paediatric patients with a tracheostomy: a multicentre epidemiological study
}

\author{
Estela Pérez-Ruiz, Pilar Caro, Javier Pérez-Frías, Maria Cols, Isabel Barrio, \\ Alba Torrent, Maria Ángeles García, Oscar Asensio, Maria Dolores Pastor, \\ Carmen Luna, Javier Torres, Borja Osona, Antonio Salcedo, Amparo Escribano, \\ Isidoro Cortell, Mirella Gaboli, Alfredo Valenzuela, Elena Álvarez, \\ Rosa Velasco and Enrique García
}

ABSTRACT: Changes in the indications for tracheostomy in children have led to the progressively greater involvement of the paediatric pulmonologist in the care of these patients. The aim of this study was to review the current profile of tracheostomised children in Spain.

We undertook a longitudinal, multicentre study over 2 yrs (2008 and 2009) of all patients aged between 1 day and 18 yrs who had a tracheostomy.

The study, involving 18 Spanish hospitals, included 249 patients, of whom $150(60.2 \%)$ were $<1 \mathrm{yr}$ of age. The main indications for the procedure were prolonged ventilation $(\mathrm{n}=156,62.6 \%)$, acquired subglottic stenosis $(n=34,13.6 \%)$, congenital or acquired craniofacial anomalies $(n=25$, $10 \%)$ and congenital airway anomalies $(n=24,9.6 \%)$. The most frequent underlying disorders were neurological diseases $(n=126,50.6 \%)$ and respiratory diseases $(n=98,39.3 \%)$. Over the 2-yr study period, 92 (36.9\%) children required ventilatory support, and decannulation was achieved in 59 (23.7\%). Complications arose in 117 patients (46.9\%). Mortality attributed to the underlying condition was $12.5 \%$ and that related directly to the tracheostomy was $3.2 \%$.

Respiratory complexity of tracheostomised children necessitates prolonged, multidisciplinary follow-up, which can often extend to adulthood.

KEYWORDS: Airway pathology, childhood disease, mechanical ventilation, paediatric tracheostomy

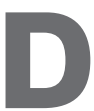
espite it being an age-old technique, paediatric tracheostomy has lately undergone a remarkable transformation, both in its indications and in the profile of the tracheostomised patient. Until some $40 \mathrm{yrs}$ ago it was considered a short-term emergency procedure, mainly to resolve acute upper airway obstruction, mostly for infectious causes, such as diphtheria, epiglotitis or laryngotracheitis. Later, the wide use of vaccination programmes, mainly against Corynebacterium diphtheriae and Haemophilus influenzae, together with the development of new anaesthetic materials and techniques to stabilise the upper airway, spectacularly reduced the number of emergent procedures in most series [1-4]. Nevertheless, despite these advances, the incidence of tracheostomy in children has not fallen; indeed, it has even risen in some hospitals [5-8]. This has been attributed to increased survival of assisted patients in paediatric and neonatal intensive care units (ICUs), with the progressive appearance of what has been termed the

For editorial comments see page 1322. "technology-dependent paediatric patient" [9], referring mainly to children on long-term assisted ventilation, or with congenital or acquired upper airway anomalies. This notable change in the indications for tracheostomy has transformed the technique into a programmed, long-term, elective procedure, just the opposite to its original indications as an emergency procedure $[2,5,10]$.

Although paediatric ear, nose and throat (ENT) services have traditionally been entrusted with the care of these patients, the changing profile of these children with tracheostomies has resulted in the paediatric pulmonologist being incorporated into the work started by the paediatric and neonatal ICUs, acting as a link between these areas and the surgical specialities, given the need for the prolonged, multidisciplinary management of these children $[6,11,12]$.

Until now, the general characteristics of tracheostomised children in Spain have remained unknown. Although each centre may have information for its

\section{AFFILIATIONS}

For author affiliations, please see the Acknowledgements section.

CORRESPONDENCE

E. Pérez-Ruiz

Hospital Carlos Haya (Materno-Infantil)

Servicio de Pediatría Neumología Pediátrica

c/Arroyo de los Ángeles s/n Málaga 29011

Spain

E-mail: estelaperezr@terra.es

Received:

Sept 212011

Accepted after revision:

March 182012

First published online:

April 102012 
own cases, no study has yet collected all the general information from the various sites. In order to obtain a more global view, therefore, the Techniques Group of the Spanish Society of Paediatric Pulmonology (SENP) undertook a multicentre study to determine the profile of the child with a tracheostomy in Spain.

\section{PATIENTS AND METHODS}

At the 2007 annual meeting of the SENP, several paediatric pulmonology representatives expressed concern about the adequate management and follow-up of children with a tracheostomy. Accordingly, it was agreed that a longitudinal registry should be compiled over 2 yrs. The SENP then published a letter explaining the aims of the study and inviting any interested hospitals to participate on its website (www.neumoped.org). The various paediatric pulmonology sections were also asked to extend the invitation to other units involved in the care of these patients, such as ENT, paediatric and neonatal ICU, and maxillofacial surgery. The target population was to be patients aged between 1 day and 18 yrs who had had a tracheostomy at some time between January 1, 2008 and December 31, 2009. A survey detailing the variables to be collected was uploaded and could then be downloaded by the various participating centres (table 1). The patients were anonymised, each one being identified by the initials of the centre and a consecutive number. Data were recorded, prospectively, on any associated complications, decannulation, start of preschool/school support and the circumstances of death if this happened during the 2-yr study period.

After receiving the completed surveys, the study co-ordinators evaluated them and then sent them to the researchers of each centre with the request that they review any missing or

\section{TABLE 1 Patient variables collected}

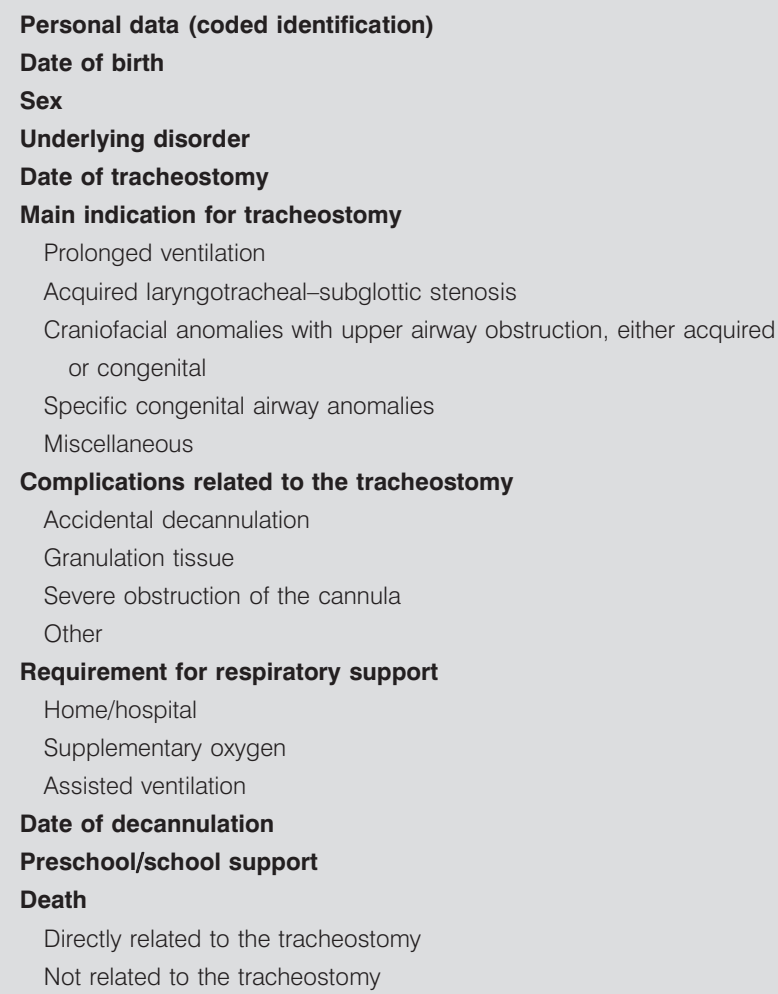

mistaken data, and give the reasons for the exclusion of any patient. Until December 31, 2009, the physicians responsible for data collection continued including patients who fulfilled the inclusion criteria and updated the clinical data of those who were already included, in the event of any variation.

After a final review at the end of the data-collection period, a database was designed (using Microsoft Office Access 2007; Microsoft Corp., Redmond, WA, USA) to process and analyse the primary variables and the secondary variables (age of each patient at the end of the study and duration of the tracheostomy). A Microsoft Excel 97-2003 spreadsheet was used to analyse the data, provide statistical evaluations and produce tables and figures.

The whole study was undertaken with the maximum confidentiality and data protection according to current legislation (Ley Orgánica 15/1999, de 13 diciembre, Protección de datos de Carácter Personal).

\section{RESULTS}

Replies were received from 22 hospitals. Four of these stated that they did not follow up tracheostomised children during the study period. The other 18, from 11 different cities in Spain, comprised the participating centres. Of the 270 patients initially recorded, the final study included 249 as 21 were excluded: 10 because they had been decannulated before the start of the study, five because they were $>18$ yrs of age at the start of the study, five because they were already included in the details reported by another hospital and one who died before the start of the study. Table 2 shows the number of patients per centre. When comparing data from the different centres we found that the number of children reported in each hospital was in proportion to the number of beds in that hospital. The large cities Madrid and Barcelona, which each have several tertiary hospitals,

\section{TABLE 2 Participating hospitals}

\begin{tabular}{lc} 
Hospitals & Patiants n (\%) \\
\hline & \\
Hospital Sant Joan de Deu, Barcelona & $30(11.6)$ \\
Hospital Val D'Hebron, Barcelona & $27(10.8)$ \\
Hospital Carlos Haya, Malaga & $25(10)$ \\
Hospital La Paz, Madrid & $24(9.6)$ \\
Hospital Niño Jesús, Madrid & $24(9.6)$ \\
Hospital Virgen de la Arrixaca, Murcia & $20(8.06)$ \\
Hospital 12 de Octubre, Madrid & $19(7.66)$ \\
Hospital Reina Sofía, Cordova & $15(6.04)$ \\
Hospital Son Dureta, Palma de Mallorca & $13(5.24)$ \\
Hospital Gregorio Marañón, Madrid & $10(4.03)$ \\
Hospital Clínico Universitario, Valencia & $10(4.03)$ \\
Hospital La Fe, Valencia & $8(3.22)$ \\
Hospital Sabadell, Barcelona & $8(3.22)$ \\
Hospital Universitario Salamanca & $6(2.4)$ \\
Hospital Virgen de las Nieves, Granada & $4(1.61)$ \\
Hospital Ramón y Cajal, Madrid & $3(1.20)$ \\
Hospital Virgen de la Salud, Toledo & $2(0.8)$ \\
Hospital General, Jaén & $1(0.40)$ \\
Total & $249(100)$ \\
\hline & \\
\hline & \\
\hline
\end{tabular}


accounted for $32 \%$ and $26 \%$, respectively, of all the patients referred. There were 150 (60.2\%) males and 99 (39.7\%) females.

The age at which tracheostomy was performed varied between the first hours of life (a male with congenital laryngeal atresia) and 17 yrs (a male who received a lung transplant due to cystic fibrosis). The median age was 6 months. Given the wide age range and the variability of the underlying disorders, the patients were grouped according to age, the largest group being that of the newborns ( $<6$ months) $(n=113,45.3 \%)$ followed by those aged 6 months -1 yr $(n=37,14.8 \%)$ (fig. 1).

Regardless of the underlying disorder, the indications leading to the procedure varied. The main reason was prolonged ventilation $(n=156,62.6 \%)$, followed at some distance by acquired subglottic stenosis $(n=34,13.6 \%)$ and others (fig. 2). Most notable among the underlying conditions were neurological disorders $(n=126$, $50.6 \%)$, with $67(53.1 \%)$ children having neuromuscular problems. Second was respiratory disorders $(n=98,39.3 \%)$, mainly bronchopulmonary dysplasia $(n=32,32.6 \%)$. Most patients, though, suffered various different processes. Of the 249 patients, $35(14 \%)$ had been born prematurely. Given that a high percentage of the children $(60.2 \%)$ were $<1 \mathrm{yr}$ of age at the time of the tracheostomy, figure 3 compares the conditions in these children versus the rest.

The duration of tracheostomy ranged from 1 day (due to perioperative mortality) to $19 \mathrm{yrs}$ (a patient with a perinatal medullary lesion). Over the 2-yr study period, decannulation was achieved in $59(23.7 \%)$ patients. The median duration of the tracheostomy in these 59 decannulated patients was 7 months (range 0.1-212 months). In the other 190 patients who remained tracheostomised due to their complex situation or who died while still cannulated, the median duration of the tracheostomy by the end of the study period was 34 months (range 0.03-236 months).

In addition to the tracheostomy, 92 (36.9\%) patients required ventilatory support during the study period, either in ICUs $(n=12,4.8 \%)$, at home $(n=79,31.7 \%)$ or both $(n=1)$. Five $(82 \%)$ of the 249 patients required a second tracheostomy. Amongst those

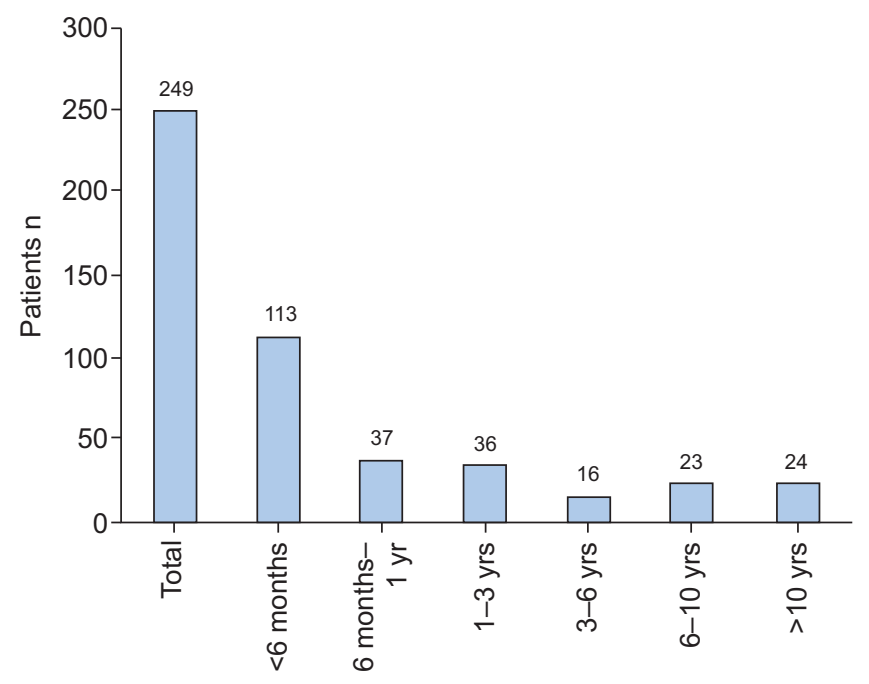

FIGURE 1. Age at which tracheostomy was performed. Stratification by age group.

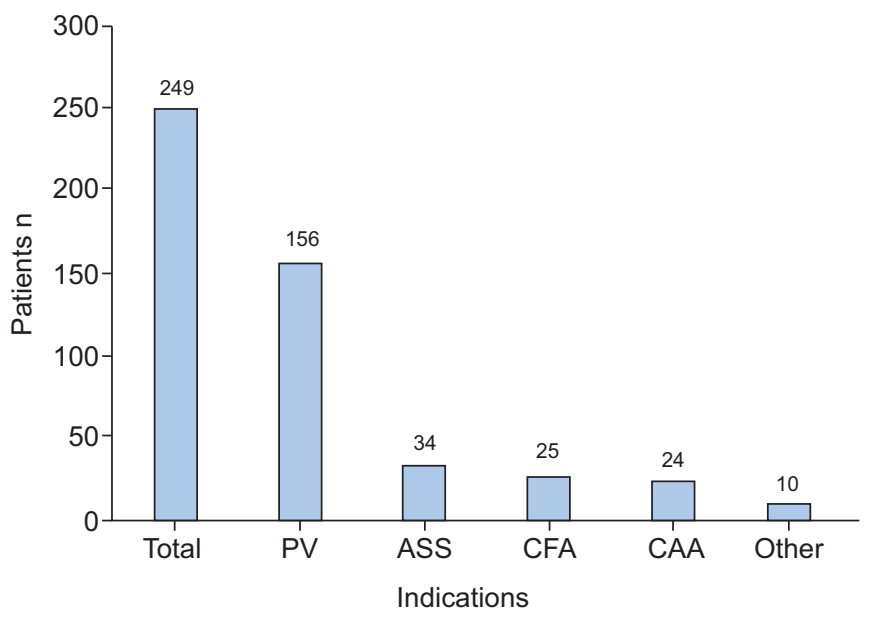

FIGURE 2. Indication for tracheostomy. PV: prolonged ventilation; ASS: acquired subglottic stenosis; CFA: craniofacial anomaly; CAA: congenital airway anomaly; Other: acquired bilateral vocal cord paralysis $(n=4)$, laryngeal papillomatosis $(n=4)$, acquired tracheo-oesophageal fistula $(n=1)$, post-operative central apnoea after cerebral tumour $(n=1)$.

children $\geqslant 4$ yrs of age (127 patients), $79.5 \%$ (101 patients) received some type of educational support while carrying a tracheostomy with or without ventilatory support, whether at the hospital, at home or even attending regular educational centres or other specialised centres for children with medical needs.

Concerning mortality and morbidity associated with the procedure, one or more complications were reported in 117 $(46.9 \%)$ patients. Figure 4 shows those during the tracheostomy procedure itself as well as those during the perioperative period or during later care, either at home or in hospital.

At the end of the study period, 218 (87.5\%) out of the initial 249 patients remained alive. Median age for the survivors was 65 months (range 2-238 months). Only one patient was lost to

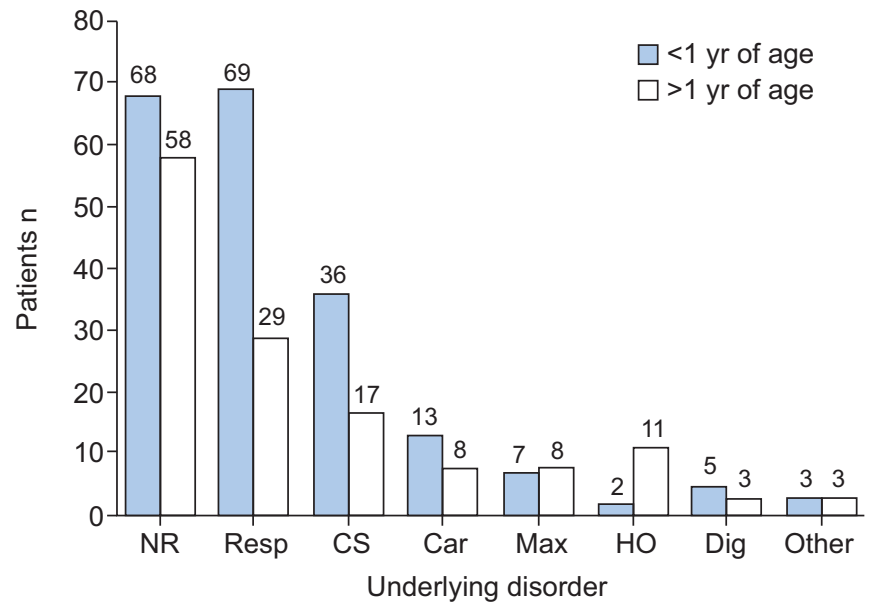

FIGURE 3. Most frequent disorders in children with tracheostomy. Comparison between those $<1 \mathrm{yr}$ and those $>1 \mathrm{yr}$ of age. Note: a patient may have more than one disorder. NR: neurological; Resp: respiratory; CS: congenital syndrome; Car: cardiopathy; Max: maxillofacial; HO: haematological-oncological; Dig: digestive; Other: patients with various comorbidities. 


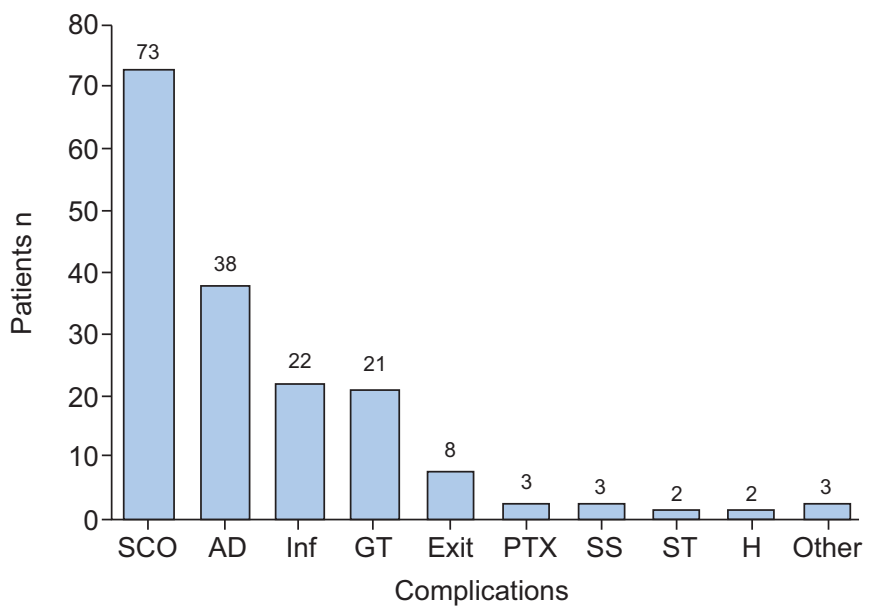

FIGURE 4. Complications associated with tracheostomy. SCO: severe cannula obstruction; AD: accidental decannulation; Inf: infection related to tracheostomy cannula; GT: important granulation tissue; Exit: death directly related to the tracheostomy; PTX: pneumothorax; SS: stomal stenosis; ST: suprastomal tracheomalacia; $H$ : haemoptysis. Other: trachea-innominate artery fistula $(n=1)$, tracheal stenosis after tracheostomy $(n=1)$ and rupture of the cannula $(n=1)$.

follow-up. Of the 31 deaths, eight were directly related to the tracheostomy; figure 5 shows the causes.

\section{DISCUSSION}

Although paediatric tracheostomy is seldom performed nowadays, it remains necessary for the management of a few "technology-dependent" children, enabling them to be discharged from the ICU [4, 12-14]. The best data regarding its true incidence stem from a study by LEWIS et al. [15], which analysed the data from 2,521 US hospitals and estimated the rate to be 6.6 children per 100,000 child-yrs during 1997. Although originally this technique was developed as an emergency procedure to solve difficult acute situations, in many settings, it has now become a programmed decision, part of a multidisciplinary approach in complex patients with chronic disorders. Indeed, CORBETT et al. [3] found that just $6 \%$ were performed for emergency airway management in a series of 122 children between 1987 and 2003.

Over recent years, numerous studies in different countries have reported the individual experience of various hospitals, with the number of patients varying from 36 to 362 over periods of time ranging from 2 to 37 yrs [3, 5, 6, 8, 9, 16-18]. Review of these reports reveals differences between the various centres. While the frequency of the technique has fallen and is almost absent in smaller hospitals, it has remained the same, or even risen slightly, in referral hospitals, due to its complexity and the need for multidisciplinary management of the children referred $[5,3,9,16]$.

Concerning the age at which the tracheostomy was performed, the study with the most patients published so far [15] indicates two peaks: $<1$ yr $(32 \%)$ and $15-18$ yrs $(10.3 \%)$, the latter mainly resulting from cranioencephalic trauma. Others have reported figures for children $<1$ yr of age of between $50 \%$ and $66 \%$ [3, 8 , 19], which is more like the $60.2 \%$ in our series. There has also been a growing trend for the procedure to be used in premature infants $(10.8 \%)[15,20]$ and in children with multiple chronic disorders or severe congenital diseases in neonatal or paediatric ICUs

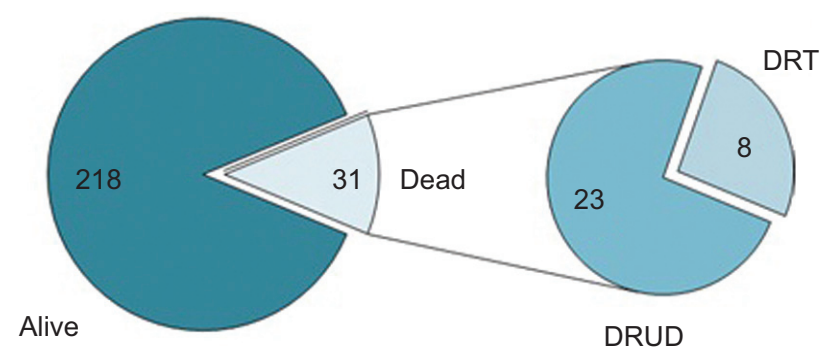

FIGURE 5. General mortality and mortality directly related to the tracheostomy. Patients alive at the end of the study: 218 (87.9\%) out of 249; patients dead at the end of the study $31(12.5 \%)$ out of 249 . DRUD: death related to the underlying disorder (23 out of $31,9.7 \%$ ); DRT: death directly related to the tracheostomy (eight out of $31,3.2 \%$ ), of which four were newborns during the immediate post-operative period (one case of suture dilaceration in a patient with subglottic congenital membrane, two cases of bilateral pneumothorax and one case due to complications with mechanical ventilation because of inadequate seal and cannula displacement). The other four patients died at home due to severe obstruction of the cannula and failure of resuscitation measures.

$[9,21,22]$. The mean age for tracheostomy in most series ranges from 3.2 to 7.8 months $[3,6,8,17]$, or around 3 yrs of age $[2,16]$. The higher mean age in our series may be due to its multicentre nature and the inclusion of referral centres for lung transplantation, which greatly increases the upper limit for the procedure. We did not detect the second peak in the incidence of tracheostomy in adolescents seen by LEWIS et al. [15], probably because the age for admission to many children's hospitals in Spain is $<16$ yrs, although chronic patients are usually followedup to a later age. As in our series, most studies have found a slightly higher frequency in males $(60-63.8 \%)[6,15,18]$, though not all (48\%) [17].

The percentage of cases due to prolonged ventilation, the main indication in our series, varies $(22-66 \%)[2,3,5,6,9,16,19]$. The low percentages in some reference centres $[5,6]$ may be related to the greater use of noninvasive ventilation or the higher number of interventional procedures in chronic obstructive airway disorders [8]. Whereas in earlier decades acquired subglottic stenosis only accounted for a small proportion of tracheostomies $(0 \%$ [23] to $5 \%$ [24]), it was the second most common indication in our series $(13.6 \%)$, a similar percentage to that found by others $[3,8,19]$, though yet others have reported much higher rates (20$36 \%)[5,6,16,17,25]$. This increase is particularly surprising, as interventional techniques now exist to resolve this condition, often without the need for tracheostomy [5, 16]. The third indication for tracheostomy in our series, upper airway obstruction secondary to craniofacial malformations or anomalies $(10 \%)$, is becoming more usual in tertiary referral hospitals (5-32.7\%) $[3,5,6,8,19]$. Finally, specific congenital airway malformations, such as tracheal stenosis, laryngo-trachea-bronchomalacia, subglottic haemangiomas or laryngo-trachea-oesophageal cleft, represented the fourth reason for tracheostomy $(9.6 \%)$. This figure is lower than that for some tertiary referral centres $(19.8 \%$ [5] or $21.4 \%$ [3]), which are seeing a notable increase as a result of the new interventional possibilities. Of interest among the other indications was that conditions associated with the classical pathogens, such as $C$. diphtheriae or epiglottis due to $H$. influenzae, have disappeared from the statistics over the last 10 yrs [3], 
although papillomavirus persists $(1.6 \%)$, as it does in other studies (1\% [5] or $2.6 \%$ [3]).

The underlying disorder (fig. 3) was more varied in the patients $<1 \mathrm{yr}$ of age, as occurred in other studies [15]. The most usual were neurological disorders, in both the younger patients ( 68 out of $159,45.3 \%$ ) and those $>1$ yr (58 out of 99, $58.5 \%)$. The high percentage of patients with neurological involvement is also noted in other series (42\%) [12]. Of note, too, in our series, was chronic respiratory involvement in children aged $\leqslant 1$ yr (69 out of $150,46 \%$ ), mainly due to bronchopulmonary dysplasia (32 out of $69,46.3 \%$ ), a disorder suffered by $32(12.8 \%)$ out of the 249 patients in this series.

Comparison of our $23 \%$ of patients who achieved decannulation with other series, which report decannulation figures of 29$52.7 \%[2,3,6,16,17,26]$, or even $75 \%$ in New Zealand [8], gives a notably lower percentage. Possible reasons for this include the shorter period of our study, the high percentage of patients with chronic neurological and respiratory indications for long-term ventilation, and the high percentage of patients $<1 \mathrm{yr}$ of age. Studies in decannulated patients indicate a mean duration of tracheostomy that can vary from 12.4 [3] to 22 months [19], depending on the underlying disorder. One of these studies found that patients $<1 \mathrm{yr}$ of age had the tracheostomy for much longer periods (829 days) than children $>1$ yr (94 days) [3]. Another study analysed the possible risk factors related to the duration of the cannulation, noting that the only determining factors were the type of disorder and the indication for tracheostomy, but not the age of the patient [27]. It is nevertheless interesting to note that despite the chronicity and complexity of the patients, $79.5 \%$ of the survivors still managed to achieve social insertion appropriate for their age via the preschool/ school support system, independently of whether they had been decannulated or were receiving ventilatory support.

The frequency of complications in our series $(46.9 \%)$ is high, as it is in other paediatric series $(51-77 \%)[1,8,26]$, in comparison with the frequency in adults. In a multicentre survey, 22\% of paediatric surgeons reported perioperative complications during the tracheostomy, leading to a change in practice by $58 \%$ of them [22]. A study of 54 patients with home mechanical ventilation (45 via tracheostomy and nine via nasal mask) reported that $66 \%$ of the severe emergencies were related to the tracheostomy [28]. Whereas infection is reported to be the main complication [26, 27], as much as $90 \%$ in some series [6], in our series it only accounted for $8.8 \%$ of complications. This difference may be related to both the shorter follow-up and the question of differentiating infection versus colonisation, under- or overestimating the true incidence. Different percentages have been reported for endotracheal granulation in various paediatric series, ranging from $12.3 \%$ to $56 \%[1,6,8]$. We only considered granulation to be a complication when it led to symptoms and was subsidiary to intervention (8.4\%). The main life-threatening complications in our study were severe obstruction of the cannula by a mucous plug $(29.3 \%)$ and accidental decannulation $(15.2 \%)$, this latter percentage being similar to that of other series $[3,6]$. The other lifethreatening complications, such as pneumothorax, subcutaneous emphysema or tracheo-innominate artery fistula, were much less usual $[2,3,19,26]$.
Mortality from the underlying condition, 9.7\% in our series, varies greatly in other studies according to the main underlying disease (6.9-39.2\%) [2, 3, 6, 8, 9, 19, 23]. Mortality directly related to the procedure, however, $(3.2 \%$ in our series), was higher than that of other studies $(0.7-3 \%)[2,3,8,18,26,29]$. Of note was the high mortality associated with the procedure in preterm infants (four in our series) (fig. 5) in comparison with other studies in which tracheostomy is presented as a safe technique at that age [20].

Our study does have certain limitations. It did not record a series of data that would, with hindsight, have proved interesting, such as the time between respiratory failure and the tracheostomy, given the high percentage of children with long-term ventilation as the main indication for elective tracheostomy. Unlike adult patients, this time is not clearly defined in children. Very variable intervals have been reported, even in the same centre (0-148 days) [6,9], which explains why the indication for tracheostomy in a child is usually personalised according to the clinical status, the endoscopic findings, the experience with noninvasive ventilation and the family circumstances $[1,12,30]$.

It would also have been of clinical interest to determine whether, before doing the tracheostomy, non-invasive ventilation had been attempted, how long the patient had been in the ICU and in hospital after the tracheostomy, the cost per patient and the percentage of readmissions after the tracheostomy, as it is easy to assume that the battle ends with the tracheostomy, when in fact a new one begins $[4,12,30]$. Indeed, in our series, $31.7 \%$ of the patients required assisted ventilation at home at the time of hospital discharge. This circumstance was also noted in a series from Houston, TX, USA (61\%), in whom $81 \%$ had additional support devices or techniques besides the tracheostomy, such as feeding tubes or central venous access [9].

Finally, although in our study we did not collect information regarding use of devices such as speaking valves or other phonatory means, we agree that early development of language can improve the social integration of these patients. Although it has been recommended that speaking valves should not be used in very small or very ill children due to the additional work of breathing, children of all ages, including infants, may be candidates for its use [11]. A new study on the use of these devices could help define indications for their use, and the true limitations in very young, small or unstable children. New surgical techniques that have been implemented in children with airway anomalies, e.g. endoscopic anterior cricoid split and balloon dilation, laryngotracheal reconstruction and posterior costal cartilage grafting or slide thyrocricotracheoplasty in subglottic stenosis, and laser therapy or tracheobronchial stents in several airway anomalies, are not reflected in this paper either, despite the positive impact that they have had in some of our patients, who could not have achieved decannulation otherwise.

\section{Conclusions}

Tracheostomy remains prevalent in tertiary children's hospitals because of the profound change in its indications. The high number of patients in our study, the different centres involved, and the study period, current and short, provide an approximate profile of the tracheostomised child in Spain: a young 
patient, generally $<1 \mathrm{yr}$ of age, with a complex underlying disorder, usually neurological or respiratory, in hospital or at home, and sometimes dependent on supplementary oxygen or assisted ventilation. These patients demand a multidisciplinary follow-up involving various specialities (ENT, paediatric surgery and neurosurgery) and paediatric areas (neonatology, intensive care and children's pulmonology), as well as additional consultations in specific paediatric areas, work with speech therapists and the work of other care institutions for family, home and school support. The complexity of the clinical status of these patients not only necessitates a prolonged follow-up during childhood, but sometimes this does not even end when they reach adolescence, the children requiring transfer to adult pulmonology and ENT services without sufficient progress in the underlying disorder to enable decannulation.

\section{STATEMENT OF INTEREST}

None declared.

\section{ACKNOWLEDGEMENTS}

Author affiliations are as follows. E. Pérez-Ruiz, P. Caro, J. Pérez-Frías: Neumología Pediátrica, Hospital Carlos Haya y Universidad de Málaga, Malaga, Spain; M. Cols: Neumología Pediátrica, Hospital San Joan de Deu, Barcelona, Spain; I. Barrio: Neumología Pediátrica, Hospital la Paz, Madrid, Spain; A. Torrent: Neumología Pediátrica y Fibrosis Quística, Hospital Vall dHebron, Barcelona, Spain; M.Á. García: Cuidados Intensivos Pediátricos, Hospital Niño Jesús, Madrid, Spain; O. Asensio: Neumología Pediátrica, Hospital Parc Taulí, Sabadell, Spain; M.D. Pastor: Neumología Pediátrica, Hospital La Arrixaca, Murcia, Spain; C. Luna: Neumología Pediátrica, Hospital 12 de Octubre, Madrid, Spain; J. Torres: Neumología Pediátrica, Hospital Reina Sofía, Cordova, Spain; B. Osona: Neumología Pediátrica, Hospital Son Espases, Palma de Mallorca, Spain; A. Salcedo: Neumología Pediátrica, Servicio de Pediatría, Hospital Gregorio Marañón, Madrid, Spain; A. Escribano: Neumología Pediátrica, Hospital Clínico, Universidad de Valencia, Spain; I. Cortell: Neumología Pediátrica, Hospital La Fé, Valencia, Spain; M. Gaboli: Neumología Infantil y Unidad de Cuidados Intensivos Pediátricos, Hospital Salamanca, Salamanca, Spain; A. Valenzuela: Neumología Pediátrica, Hospital Virgen de las Nieves, Granada, Spain; E. Álvarez: Cuidados Intensivos Pediátricos, Hospital Ramón y Cajal, Madrid, Spain; R. Velasco: Neumología Pediátrica, Hospital Virgen de la Salud, Toledo, Spain; E. García: Neumología Pediátrica, Hospital General de Jaén, Jaén, Spain.

\section{REFERENCES}

1 Davis GM. Tracheostomy in children. Paediatr Respir Rev 2006: Suppl. 7, 206s-209s.

2 Carron JD, Derkay CS, Strope GL, et al. Pediatric tracheotomies: changing indications and outcomes. Laryngoscope 2000; 110: 1099-1104.

3 Corbett HJ, Mann KS, Mitrab I, et al. Tracheostomy - a 10-year experience from a UK pediatric surgical center. J Pediatr Surg 2007; 42: 1251-1254.

4 Fields AI. Pediatric tracheostomy: the great liberator or the last battlefield. Pediatr Crit Care Med 2008; 9: 126-127.

5 Hadfield PJ, Lloyd-Faulconbridge RV, Almeyda J, et al. The changing indications for paediatric tracheostomy. Int $J$ Pediatr Otorhinolaryngol 2003; 67: 7-10.
6 Al-Samri M, Mitchell I, Drumond DS, et al. Tracheostomy in children: a population-based experience over 17 years. Pediatr Pulmonol 2010; 45: 487-493.

7 Özmen S, Özmen OA, Ünal OF. Pediatric tracheostomies: a 37year experience in 282 children. Int J Pediatr Otorhinolaryngol 2009; 73: 959-961.

8 Mahadevan M, Barber C, Salked L, et al. Pediatric tracheotomy: 17 year review. Int J Pediatr Otorhinolaryngol 2007; 71: 1829-1833.

9 Graf M, Montagnino BA, Hueckel R, et al. Pediatric tracheostomies: a recent experience from one academic center. Pediatr Crit Care Med 2008; 9: 96-100.

10 Trachsel D, Hammer J. Indications for tracheostomy in children. Paediatr Respir Rev 2006: Suppl. 7, 162s-168s.

11 Eber E, Oberwaldner B. Tracheostomy care in the hospital. Paediatr Respir Rev 2006: Suppl. 7, 175-184.

12 Graft JM, Montagnino BA, Huekel R, et al. Children with new tracheostomies: planning for family education and common impediments to discharge. Pediatr Pulmonol 2008; 43: 788-794.

13 Cohen E, Kuo DZ, Agrawal R, et al. Children with medical complexity: an emerging population for clinical and research initiatives. Pediatrics 2011; 127: 529-538.

14 Principi T, Morrison GC, Matsui DM, et al. Elective tracheostomy in mechanically ventilated children in Canada. Intensive Care Med 2008; 34: 1498-1502.

15 Lewis CW, Carron JD, Perkins JA, et al. Tracheotomy in pediatric patients. A national perspective. Arch Otolaryngol Head Neck Surg 2003; 129: 523-529.

16 Butnaru CS, Colreavy MP, Ayari S, et al. Tracheotomy in children: evolution in indications. Int J Pediatr Otorhinolaryngol 2005; 70: 115-119.

17 Vanker A, Kling S, Booysen JR, et al. Tracheostomy home care: in a resource-limited setting. Arch Dis Child 2012; 97: 121-123.

18 Alladi A, Rao S, Das K, et al. Pediatric tracheostomy: a 13-year experience. Pediatr Surg Int 2004; 20: 695-698.

19 Parrilla C, Scarano E, Guidi ML, et al. Current trends in paediatric tracheostomies. Int J Pediatr Otorhinolaryngol 2007; 71: 1563-1567.

20 Pereira KD, MacGregor AR, Mitchell RB. Complications of neonatal tracheostomy: a 5-year review. Otolaryngol Head Neck Surg 2004; 131: 810-813.

21 Fraga JC, de Souza JC, Kruel J. Pediatric tracheostomy. J Pediatr (Rio J) 2009; 85: 97-103.

22 Ruggiero FP, Michele M, Carr MM. Infant tracheotomy. Arch Otolaryngol Head Neck Surg 2008; 134: 263-267.

23 Carter P, Benjamin B. Ten-year review of paediatric tracheotomy. Ann Otol Rhinol Laryngol 1983; 92: 398-400.

24 Wetmore RF, Handler SD, Potsic WP. Paediatric tracheostomy: experience during the past decade. Ann Otol Rhinol Laryngol 1982; 91: 628-632.

25 Midwinter KI, Carrie S, Bull PD. Pediatric tracheostomy: Sheffield experience 1979-1999. J Laryngol Otol 2002; 116: 532-535.

26 Carr MM, Poje CP, Kingston L, et al. Complications in pediatric tracheostomies. Laryngoscope 2001; 111: 1925-1928.

27 Leung R, Berkowitz RG. Decannulation and outcome following pediatric tracheostomy. Ann Otol Rhinol Laryngol 2005; 114: 743-748.

28 Reiter K, Pernath N, Pagel P, et al. Risk factors for morbidity and mortality in pediatric home mechanical ventilation. Clin Pediatr (Phila) 2011; 50: 237-243.

29 Zia S, Arshad M, Nazir Z, et al. Pediatric tracheostomy: complications and role of home care in a developing country. Pediatr Surg Int 2010; 26: 269-273.

30 Kun SS, Davidson-Ward SL, Hulse LM, et al. How much do primary care givers know about tracheostomy and home ventilator emergency care? Pediatr Pulmonol 2010; 45: 270-274. 